\title{
Harlequin Fetus in a Twin Pregnancy: An Extremely Rare Presentation
}

\author{
Arfan ul Bari and Syed Umair Ahmed Najmi \\ Department of Dermatology, Combined Military Hospital, Malir Cantt, Karachi, Pakistan
}

\begin{abstract}
Harlequin ichthyosis $(\mathrm{HI})$ is a rare fatal type of congenital ichthyosis with autosomal recessive inheritance pattern. We report an uncommon presentation of a case of $\mathrm{HI}$ in a neonate, who was the second outcome of the dizygotic twin pregnancy of a Pakistani female. Whilst the first twin remained alive and healthy, $\mathrm{HI}$ baby was not able to survive beyond the third day of her life. Although very few cases of $\mathrm{HI}$ from Pakistan have been reported in the past; but this case being one of the outcomes of twin pregnancy is unique in its presentation; and to the best of authors' knowledge, has not been reported earlier.
\end{abstract}

Key Words: Harlequin ichthyosis, Autosomal recessive, Congenital ichthyosis, Twin pregnancy.

How to cite this article: Bari AU, Najmi SUA. Harlequin Fetus in a Twin Pregnancy: An Extremely Rare Presentation. J Coll Physicians Surg Pak 2020; 30(06):652-654 https://doi.org/10.29271/jcpsp.2020.06.652.

\section{INTRODUCTION}

The term ichthyosis generally applies to a group of heterogeneous genetic disorders of abnormal keratinisation and the term autosomal recessive congenital ichthyosis ( $\mathrm{ARCI}$ ) encompasses non-syndromic forms of $\mathrm{ARCl}$ without blistering tendency. The spectrum includes harlequin ichthyosis ( $\mathrm{HI})$, lamellar ichthyosis (LI), congenital ichthyosiform erythroderma (CIE), bathing-suit ichthyosis (BSI), and Collodion baby. ${ }^{1} \mathrm{HI}$ is associated with mutations in the gene ABCA12 located on chromosome 2 (2q34). ABCA12 is a keratinocyte transmembrane lipid transporter considered essential for the transport of lipids to form extracellular lipid layer in the stratum corneum. ${ }^{2}$ Mutation in the gene hampers the transfer of lipids into lamellar granules giving rise to formation of abnormal cytoplasmic vacuoles in keratinocytes. Resultantly, the stratum corneum becomes remarkably thickened and is not able to desquamate normally. Histologically, lesions are characterised by massive hyperkeratosis (with lipid deposits), acanthosis, papillomatosis and normal or absent granular cell layer. Keratinocytes display hyper proliferative keratins $\mathrm{K} 6$ and K16 and an inability to convert profilaggrin to filaggrin. ${ }^{1-3}$ Clinically, $\mathrm{HI}$ babies are mostly premature covered all over with fissured "armor-plated" skin, that is considerably taut causing difficulty in movements and inability to drink and breathe.

Correspondence to: Dr. Arfan ul Bari, Department of Dermatology, Combined Military Hospital, Malir Cantt, Karachi, Pakistan

E-mail: albariul@gmail.com

Received: May 29, 2019; Revised: August 30, 2019;

Accepted: September 12, 2019

DOI: https://doi.org/10.29271/jcpsp.2020.06.652
Additional findings include bilateral ectropion, eclabium, open O-shaped mouth, hypoplastic fingers and hyperkeratotic skin, resulting in ears lacking retroauricular folds. Patients are at high risk for auto amputation of digits, hypo or hyperthermia, electrolyte imbalances, respiratory distress and dreadful skin and lung infections, which are the leading causes of mortality in the early days of life. Fatality is very high and most affected neonates are unable to survive beyond the first week of life. Management remains palliative but with good neonatal care and early introduction of oral retinoid, survival rates may significantly improve. Recurrence rate in the subsequent pregnancy is $25 \%$; therefore, genetic counselling should be done for these cases. ${ }^{4,5}$

We report a rare case of $\mathrm{HI}$ in a neonate, who was the second twin of the dizygotic twin pregnancy. The neonate survived for 2 days and 15 hours after palliative care in neonatal intensive care unit. The other twin was completely normal. Our basic objective to present this case is to highlight its extreme rarity in dizygotic twin pregnancy which needs timely and appropriate management to avert and combat the potential complications associated with it.

\section{CASE REPORT}

A 26-year pregnant woman was referred from secondary care health centre to this Hospital for management and care of her fourth pregnancy with twins. She already had three normal live births. Antenatal and anomaly ultrasound scans confirmed twins (diamniotic, mono-chorionic) and was unremarkable for any gross fetal anomalies. Gestational age was approximately 36 weeks and 1 day, based on both last menstrual period (LMP) and the scans. Elective lower segment cesarean section (LSCS) was planned and both twins were delivered. The first twin was normal (Figure 1). However, the second twin had the typical features of $\mathrm{HI}$ with thick porcelain white skin with intervening deep creases covering the whole body (Figure 2). Parents had 
consanguineous marriage but there was no family history ofany genetic diseases in the family. Three siblings were also normal. On physical examination, the baby was covered and encased with massive thick polygonal armor-like plates of statrum corneum with fissures extending deep into the skin (Figure 3). There was marked bilateral ectropion with immature eyes, rudimentary auricles and eclabium with persistent open mouth (Figure 3). All the limbs and extremities were hypoplastic and held in a semi-flexed position due to extremely rigid and stretched skin (Figure 3). The cry was also very characteristic.

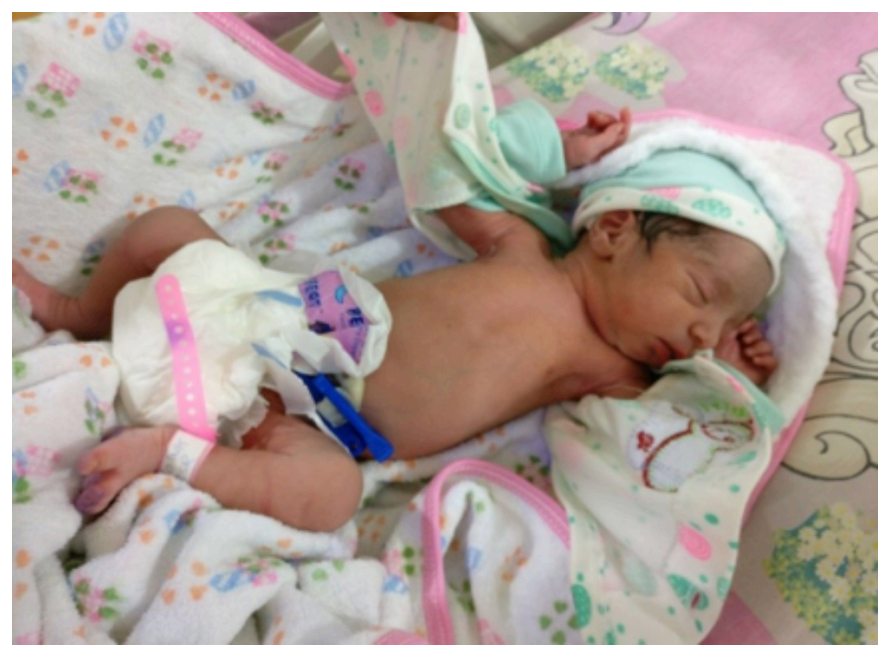

Figure 1: Normal healthy newborn of twins delivered first.

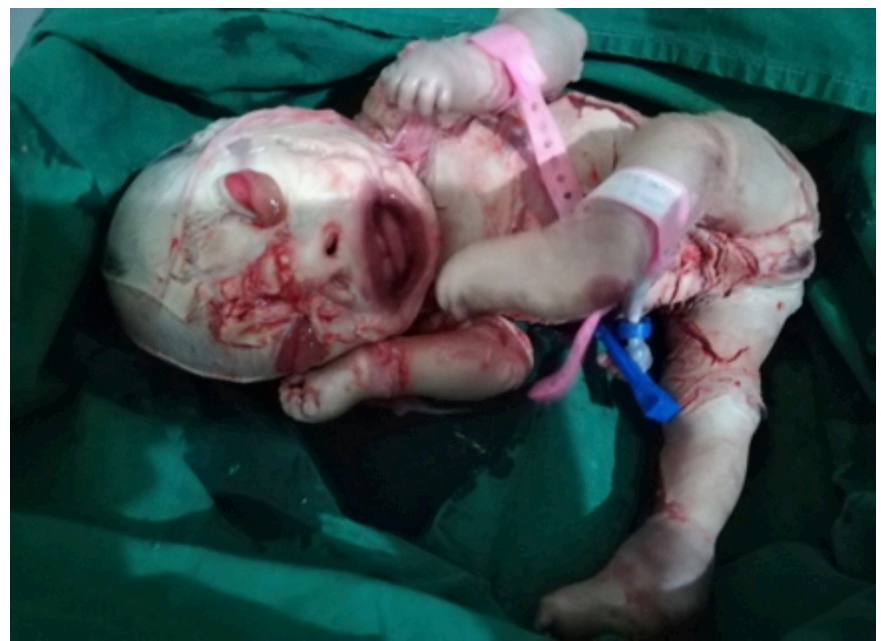

Figure 2: Enormously thick porcelain-like skin covering the body like armor with deep creases all over the body, severe ectropion, rudimentary ears, eclabium with open mouth.

The baby was nursed in the NICU and placed in a humidified incubator with cardiorespiratory monitoring. Parenteral nutrition was initiated on the day one as the baby was unable to suck and inability of a nasogastric tube to be inserted due to blocked nostrils. Antibiotic coverage and electrolytes were initiated. Skin care with bland emollients and wet dressings was started and oral retinoid therapy was planned on stabilization after few critical initial days. Unfortunately, neonate could not survive long and died on the third day of delivery.

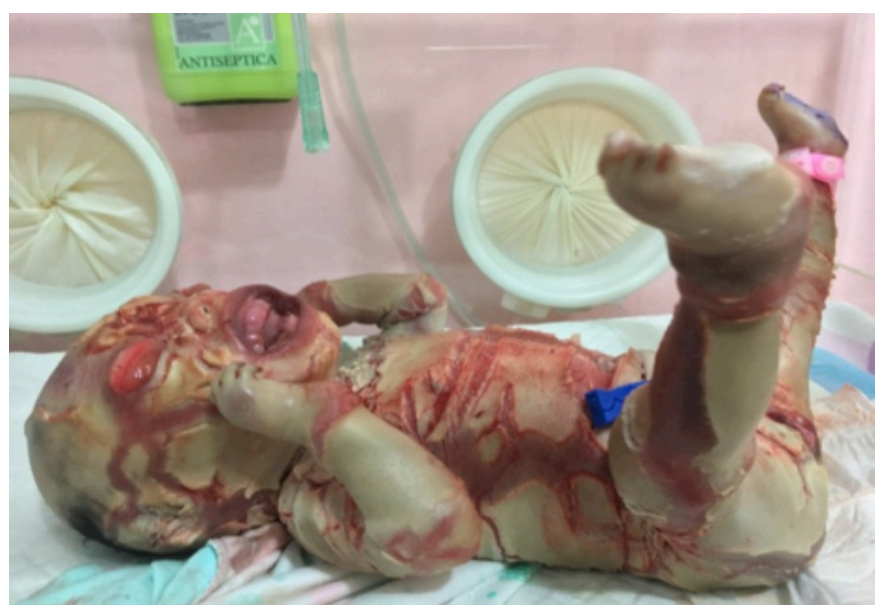

Figure 3: Large polygonal plates of skin with deep fissures leading to bleeding with limbs in rigid semi-flexion.

\section{DISCUSSION}

The term harlequin is derived from the general facial appearance and the triangular and diamond shaped pattern of hyperkeratosis. $\mathrm{HI}$ is the least common and most severe type of $\mathrm{ARCl}$ with lethality around $44 \%$. Its incidence is about 1 in 300,000 births. ${ }^{4,5}$ The firstcase of HI was reported in 1750 , and it was diagnosed antenatally for the first time in $1983 .{ }^{6,7}$ Scanning features that help in antenatal clinical diagnosis include; polyhydramnios, echogenic amniotic fluid, fetal growth retardation, ectropion, eclabium, flat nose, wide open mouth, rudimentary ears, flexion deformities of extremities, mottled, breeched/deeply fissured skin of the face and limbs and hyper-flexed fingers and toes. ${ }^{5-7}$ Clinically, suspected cases during antenatal examinations can further be confirmed by chorionic villus sampling or amniocentesis (DNA-based diagnosis) and through fetal skin biopsy (electron microscopy). ${ }^{3}$ Approximately 200 cases have so far been reported throughout the world. ${ }^{5}$ Although most of these cases have been described as isolated ones; but there has been few reports of $\mathrm{Hl}$ occurring in siblings, including one case of twin pregnancy. ${ }^{8-10}$

The present case is distinctive and unique of its kind as one fetus out of twins presented as fatal case of $\mathrm{HI}$ and the other as normal healthy newborn. In the only other reported case of HI occurring in twin pregnancy, both fetuses were equally affected and could not survive beyond few days. ${ }^{9}$ In this case, affected fetus expired on third day; while the normal one is living and healthy. To the very best of authors' knowledge, no such case has been reported earlier. Typically, $\mathrm{HI}$ cases are born prematurely leaving the infants at the risk for complications from early delivery; but in this case, it was almost term pregnancy where elective LSCS was performed without any peroperative or postoperative complications.

This fatal condition can be diagnosed antenatally by observing characteristic scanning features; and proactive management can appropriately avert and combat associated potential complications. 


\section{PATIENTS' CONSENT:}

Informed consents were obtained from parents of all patients to publish the data concerning this case.

\section{CONFLICT OF INTEREST:}

There is no conflict of interest to declare by principal author or co-author.

\section{AUTHORS' CONTRIBUTION:}

AUB: Actively managed the case, conceptualised for publication, arranged data, prepared manuscript and revised according to the suggestions of reviewers.

SUN: Involved in all stages of management of the case, gathering relevant data, preparation of manuscript and its revision.

\section{REFERENCES}

1. Takeichi T, Akiyama M. Inherited ichthyosis: Non-syndromic forms. J Dermatol 2016; 43(3):242-51.

2. Thomas AC. Premature terminal differentiation and a reduction in specific proteases associated with loss of abcal2 in harlequin ichthyosis. Am J Pathol 2009; 174(3): 970-8.

3. Jilumudi UB. Harlequin Ichthyosis: A medico legal case report \& review of literature with peculiar findings in autopsy. J Forensic Leg Med 2012; 19(6):352-4.

4. Rajpopat S, Moss C, Mellerio J, Vahlquist A, Ganemo A, Hellstrom-Pigg $M$, et al. Harlequin ichthyosis: A review of clinical and molecular findings in 45 cases. Arch Dermatol 2011; 147(6):681-6.

5. Shruthi B, Nilgar BR, Dalal A, Limbani N. Harlequin ichthyosis: A rare case. Turk J Obstet Gynecol 2017; 14(2): 138-40.

6. Nayak S, Dash SP, Khatua M. Fetal harlequin ichthyosis - A case report. J Dent Medi Sci 2015; 14:81-6.

7. Bongain A, Benoit B, Ejnes L, Lambert JC, Gillet JY. Harlequin fetus: Three- dimensional sonographic findings and new diagnostic approach. Ultrasound Obstet Gynecol 2002; 20(1):82-5.

8. Unamuno P, Pierola JM, Fernandez E, Roman C, Velasco JA. Harlequin foetus in four siblings. Br J Dermatol 1987; 116(4):569-72.

9. Reyes Bou Z, García I, García L, Santiago Borrero PJ, Valcárcel M. First case of dizygotic twins with lamellar ichthyosis in Puerto Rico. Bol Asoc Med P R 2012; 104(1): 40-2.

10. Habib A, Pasha W, Raza N, Hameed A. Harlequin ichthyosis in two siblings. J Coll Physicians Surg Pak 2011; 21(8): 503-5. 